\title{
OPEN Field-deployable, rapid diagnostic testing of saliva for SARS-CoV-2
}

\author{
Shan Wei ${ }^{1,4}$, Hemant Suryawanshi ${ }^{1,4}$, Alexandre Djandji ${ }^{1}$, Esther Kohl ${ }^{1}$, Stephanie Morgan ${ }^{1}$, \\ Eldad A. Hod ${ }^{1,2}$, Susan Whittier ${ }^{1,2}$, Kevin Roth ${ }^{1,2}$, Raymond Yeh ${ }^{1}$, Juan Carlos Alejaldre ${ }^{1,2}$, \\ Elaine Fleck ${ }^{1,2}$, Stephen Ferrara ${ }^{1,2}$, Daniel Hercz ${ }^{3}$, David Andrews ${ }^{3}$, Lilly Lee ${ }^{3}$, \\ Kristopher A. Hendershot ${ }^{3}$, Joshua Goldstein ${ }^{3}$, Yousin Suh ${ }^{1}$, Mahesh Mansukhani ${ }^{1,2}$ \& \\ Zev Williams ${ }^{1,2} \bowtie$
}

To safely re-open economies and prevent future outbreaks, rapid, frequent, point-of-need, SARSCoV-2 diagnostic testing is necessary. However, existing field-deployable COVID-19 testing methods require the use of uncomfortable swabs and trained providers in PPE, while saliva-based methods must be transported to high complexity laboratories for testing. Here, we report the development

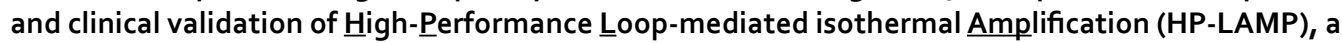
rapid, saliva-based, SARS-CoV-2 test with a limit of detection of 1.4 copies of virus per $\mu$ l of saliva and a sensitivity and specificity with clinical samples of $>96 \%$, on par with traditional RT-PCR based methods using swabs, but can deliver results using only a single fluid transfer step and simple heat block. Testing of 120 patient samples in 40 pools comprised of 5 patient samples each with either all negative or a single positive patient sample was $100 \%$ accurate. Thus, HP-LAMP may enable rapid and accurate results in the field using saliva, without need of a high-complexity laboratory.

Frequent, rapid, sensitive, and accurate COVID-19 testing that can be scaled and deployed in the field is critical for controlling the ongoing pandemic and preventing future outbreaks ${ }^{1-3}$. However, existing methods either use nasal/nasopharyngeal swabs, which require the use and exposure of trained personnel and personal protective equipment (PPE) and are less conducive to frequent testing in the general population, or use saliva but must be transported to high complexity laboratories for testing ${ }^{3-5}$. The ability to perform testing frequently and in the field with results available rapidly but with a low limit of detection is important because it permits self-isolation and quarantine early in the course of infection and can serve a "gating" function to limit entry of infected individuals into a high-risk environment, thereby preventing asymptomatic transmission ${ }^{6-10}$.

Reverse Transcription Loop-mediated isothermal Amplification (RT-LAMP), is a targeted isothermal nucleic acid amplification method that utilizes a combination of 2-3 primer sets and a DNA polymerase with high strand displacement activity ${ }^{11}$. While RT-LAMP has been used for SARS-CoV-2 detection by several groups ${ }^{6,12-14}$, these methods require a prior extraction step or lengthy sample treatment (which makes it difficult to deploy in the field), multiple fluid transfer steps, or lack the accuracy and limit of detection necessary for clinical implementation, and are therefore not suitable for clinical testing outside of a laboratory. Here we report the development and initial validation of a SARS-CoV-2 detection assay based on RT-LAMP, but with significant modifications made to enable detection of single-copy levels of virus in $<30$ min directly from heat-inactivated saliva using only a single fluid transfer step and simple heat block with a simple colorimetric readout that can be interpreted with the unaided eye. We term the new assay High-Performance LAMP (HP-LAMP). A diagram illustrating the principle of HP-LAMP is shown in Fig. 1 and the workflow is illustrated in Fig. 2A.

To develop HP-LAMP, we first designed novel primers for targeting the SARS-CoV-2 virus. Existing primers used for RT-PCR and LAMP based nucleic acid testing of SARS-CoV-2 target the GC-rich regions located at the $5^{\prime}$ and $3^{\prime}$ ends of the virus. However, because salivary exonucleases degrade viral RNA from the ends, we designed our primers to target the central portion of the virus that would be better protected. We designed eight sets of six LAMP primers targeting SARS-CoV-2 reference genome (NC_045512.2) (Fig. 2B, Figure S1A). The central region of SARS-CoV-2 genome is GC-poor (AT-rich), making it difficult to select primer candidates across the genome with optimal annealing temperatures when following standard parameters for primer design. Therefore, we designed the primers to permit large primer-mediated loop-structures while ensuring that the primers did not form stable secondary structures or self-dimerize. We tested these primer sets along with previously

${ }^{1}$ Columbia University Irving Medical Center, New York, NY, USA. ${ }^{2}$ New York Presbyterian Hospital, New York, NY, USA. ${ }^{3}$ Jackson Memorial Hospital, Miami, FL, USA. ${ }^{4}$ These authors contributed equally: Shan Wei and Hemant Suryawanshi. ${ }^{\boxplus}$ email: zw2421@cumc.columbia.edu 


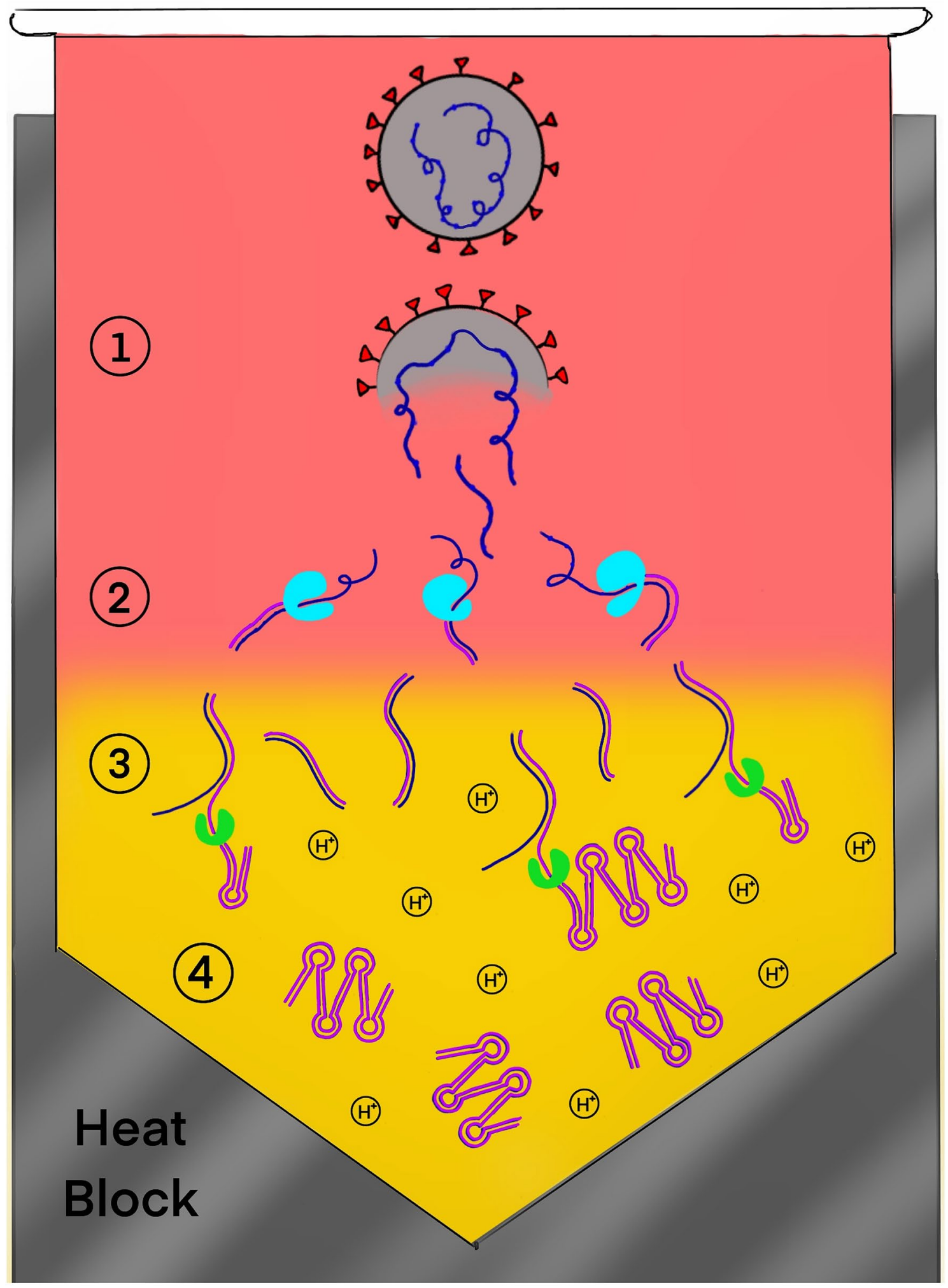

Figure 1. Diagram illustrating the principle of the HP-LAMP assay. Four steps occur within a single microcentrifuge tube containing the HP-LAMP cocktail. These steps consist of (1) Heat-mediated release of the single-stranded RNA virus (blue) from the virion, (2) Reverse transcription of the single-stranded viral RNA to generate cDNA (purple), (3) Loop-mediated amplification (LAMP) resulting in a decrease in $\mathrm{pH}$, (4) Color change caused by decreased $\mathrm{pH}$. The addition of RNA, DNA and RNAse inhibitors to the HP-LAMP cocktail inhibits degradation of the viral RNA by RNAses naturally found in saliva. 
A

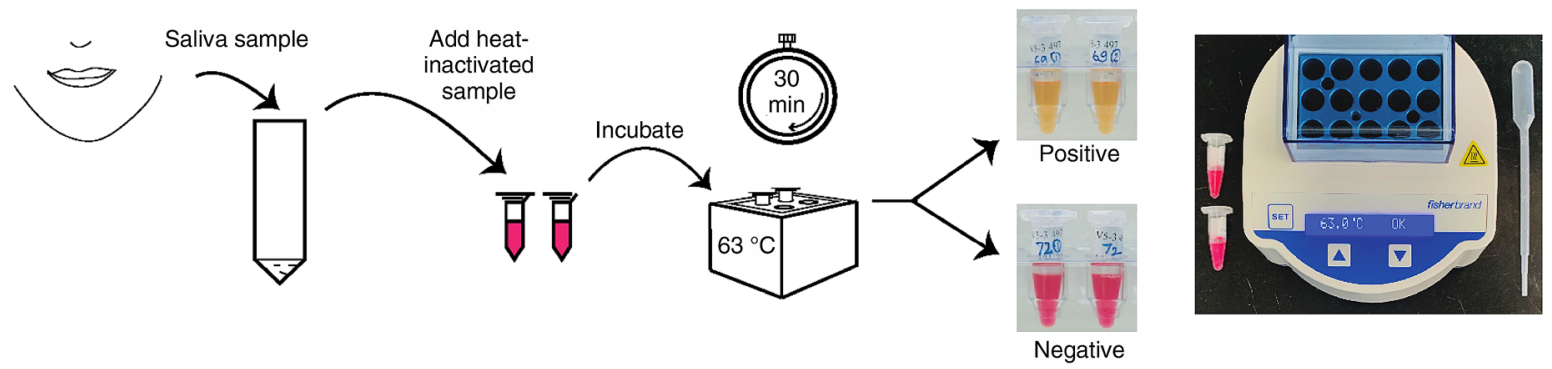

B

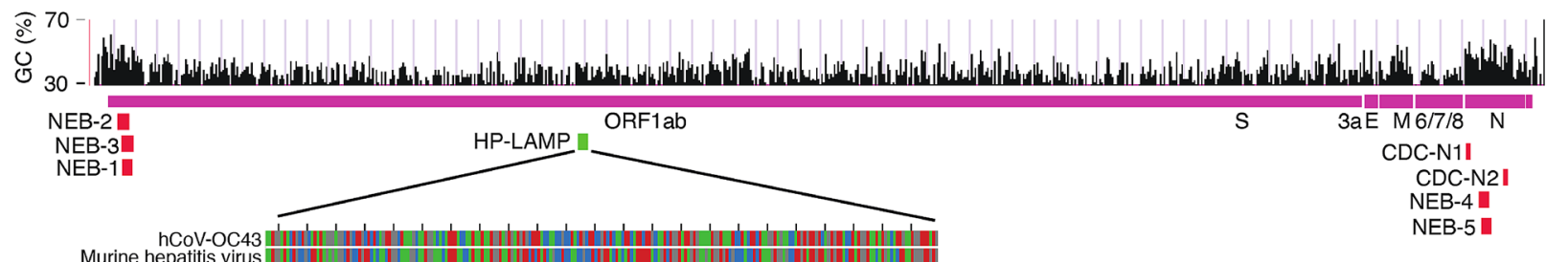

Murine hepatitis virus

hCoV-HKU1

hCoV-NL63

MERS-COVEngland 1 M

SARS-CoV Tor2 SARS-CoV-2
SARS-CoV-2 isolate

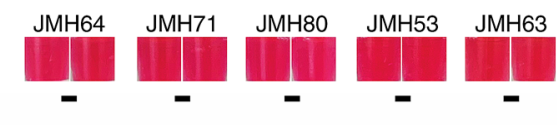

C
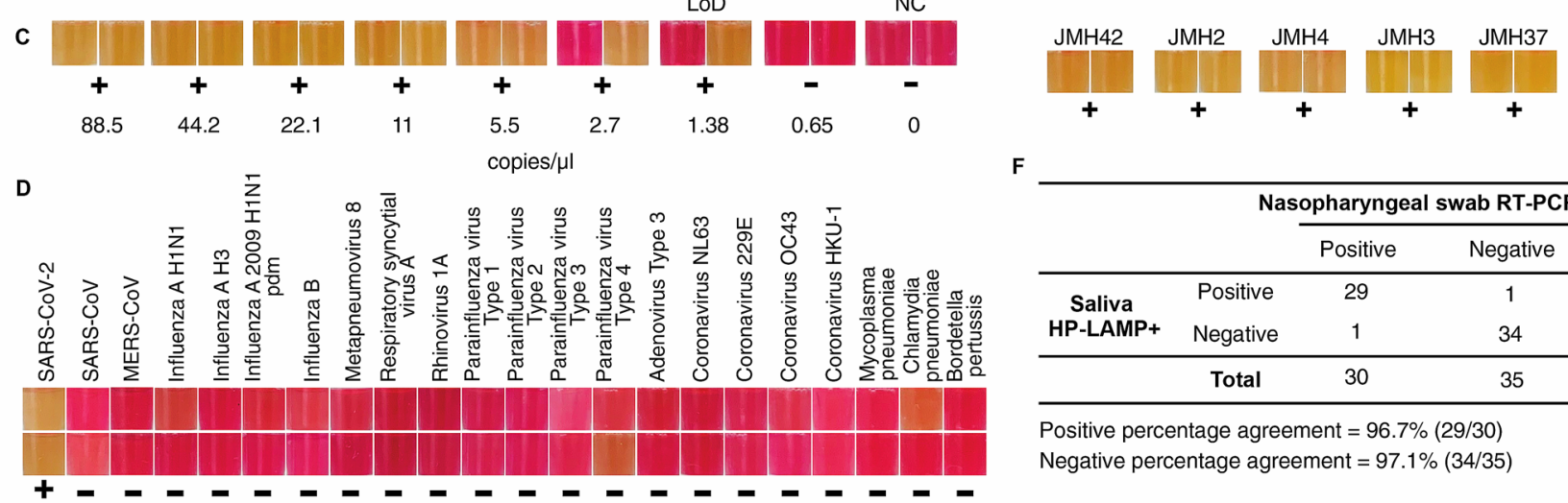

$\mathbf{F}$

\begin{tabular}{cccc}
\hline & \multicolumn{3}{c}{ Nasopharyngeal swab RT-PCR } \\
\cline { 3 - 4 } & & Positive & Negative \\
\hline \multirow{2}{*}{ Saliva } & Positive & 29 & 1 \\
HP-LAMP+ & Negative & 1 & 34 \\
\hline & Total & 30 & 35 \\
\hline
\end{tabular}

Positive percentage agreement $=96.7 \%(29 / 30)$

Negative percentage agreement $=97.1 \%(34 / 35)$

Figure 2. (A) HP-LAMP assay workflow. Heat-inactivated saliva $(5 \mu \mathrm{l})$ was added to each of the two $1.5 \mathrm{ml}$ microcentrifuge tubes pre-filled with the reaction mixture, incubated at $63^{\circ} \mathrm{C}$ for $30 \mathrm{~min}$ and then visualized for colorimetric change (yellow = positive; red=negative). At least one out of two tubes must turn yellow to interpret the assay result as positive. The minimum equipment needed to run the assay is a disposable transfer pipette, 2 heat blocks, and microcentrifuge tubes prefilled with reaction mixture. No prior RNA extraction or treatment is required. (B) Genome map showing targeted region of primers used for HP-LAMP in green. Locations of primers and probes from the CDC 2019-nCoV Real-Time RT-PCR Diagnostic Panel/New York SARS-CoV-2 Real-time RT-PCR Diagnostic Panel, and the New England Biolabs' (NEB) SARS-CoV-2 assay are indicated in Red. Whereas previously used primers and probes targeted the GC-rich regions located at the $5^{\prime}$ and $3^{\prime}$ ends of the viral RNA, the primers used for HP-LAMP target the central portion of the viral RNA, which is better protected from digestion by salivary exonucleases. The targeted region of the HP-LAMP primer set with alignments of other Betacoronavirus genomes are featured ${ }^{29,15}$. Each nucleotide is shown (A: green; G: gray; T: red; C: blue). The percentage of GC-content across the genome is indicated (http://genome.ucsc.edu) ${ }^{30,16}$. (C) Determining the limit of detection (LoD) of the HP-LAMP assay. The concentrations indicated show copies of heat-inactivated SARS-CoV-2 per $\mu$ l of saliva. NC= negative control with no SARS-CoV-2 added. ' + ' = Positive HP-LAMP result; '-' = Negative HP-LAMP result. The color of each box is taken directly from its corresponding reaction tube. (D) Cross-reactivity of HP-LAMP assay on common pathogens. Inactivated known respiratory pathogens $(n=21)$ along with inactivated SARS-CoV-2 virus were tested using HP-LAMP assay. All pathogens showed negative detection results in HP-LAMP assay, expect for SARS-CoV-2 virus. (E) Representative results of HP-LAMP testing on clinical samples. (F) Table shows positive and negative percentage agreement of HP-LAMP for detection of SARS-CoV-2 in saliva compared with nasopharyngeal swab RT-PCR results.

published primer sets (Table S1) ${ }^{12,17}$, using serial dilutions of 500 to 0.5 copies of SARS-CoV-2 RNA standard spiked into a $25 \mu \mathrm{l}$ standard RT-LAMP reaction (Figure S1A). The in-house designed primer set V5 detected $10^{\circ}$ to $10^{-1}$ copies level viral RNA in water, representing a 10 - to 100 -fold improvement in sensitivity and equivalent 
specificity compared with previously published primer sets (Figure S1A). In-silico inclusivity analysis of primer set V5 performed by aligning all primer sequences against all $(\mathrm{n}=16,453)$ complete SARS-CoV-2 genomic sequences deposited in the NCBI Virus database on September 15, 2020 showed a 100\% match for the ORF1ab gene was found for $98.8 \%$ of SARS-CoV-2 strains $(n=16,264), 1$ mismatch was found to $1.2 \%(n=182)$, and 2 mismatches were found to $0.04 \%(n=7)$ of strains deposited in the NCBI Virus database ${ }^{15}$, respectively (Table S2). No instances of more than two mismatches were found. In silico cross-reactivity/exclusivity was performed by aligning the V5 primer sequences against sequences of 32 common viruses as well as coronaviruses related to SARS-CoV-2. Both the Forward Inner Primer (FIP) and the Backward Inner Primer (BIP) consist of 2 sections of non-continuous genomic sequences and were aligned separately to increase the sensitivity of alignment of crossreactivity. In total, 6 primers corresponding to 8 sections of virus genome were assessed in silico for potential cross-reactivity against 32 common respiratory pathogens including six other human coronaviruses (SARS-CoV, MERS-CoV, HCoV-HKU-1, HCoV-NL63, HCoV-OC43 and HCoV-229E) (Table S3) ${ }^{17}$. None of the pathogens tested have a match against the total sequence length of the SARS-CoV-2 primers greater than the recommended threshold of $80 \%$, except for SARS-CoV virus. The greatest percentage match is $92.0 \%$ on part $1(\sim 50 \%)$ of the

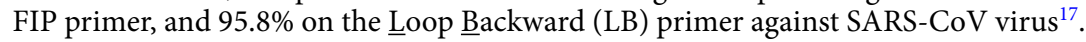

Next, we systematically modified the RT-LAMP reaction conditions to improve performance. We found that sensitivity and specificity of the assay could be markedly improved by adding carrier DNA, carrier RNA, and RNase inhibitors, as well as by increasing the reaction volume and introducing a heat-inactivation step (Figure S1B-J). Because of the risk that carry-over product from prior samples could cross contaminate a new sample and lead to false-positive results, we added Deoxyuridine Triphosphate (dUTP) and Antarctic Thermolabile uracil-DNA N-glycosylase (UDG) to our reaction mixture to incorporate dUTP into the HP-LAMP product and digest the HP-LAMP carry-over ${ }^{17}$.

To determine the limit of detection (LoD) of HP-LAMP, twofold serial dilutions of intact virus were spiked into negative saliva in concentrations ranging from 88.5 to 0.69 copies/ $\mu \mathrm{l}$ of saliva (Fig. $2 \mathrm{C}$ ). At the LoD of 1.38 copies/ $\mu$ l of saliva, $19 / 20$ replicates $(95 \%)$ were positively detected. At $2 \times \operatorname{LoD}(2.7$ copies/ $\mu$ l of saliva), 20/20 replicates (100\%) were detected (Figure S2). This LoD is comparable to other U.S. Food and Drug Administration (FDA) Emergency Use Authorization (EUA) authorized swab- and saliva-based tests that must be run in centralized high complexity laboratories, including swab-based assays, such as LabCorp's COVID-19 RT-PCR test ( 15.625 copies/reaction), the Centers for Disease Control and Prevention (CDC) 2019-nCoV Real-Time RT-PCR panel $\left(\sim 10^{0} \text { to }-0.5 \text { copies/ } \mu \mathrm{L}\right)^{18,19}$, SalivaDirect ( 6 copies/ $\left.\mu \mathrm{L}\right)$, Fluidigm Corporation's Advanta Dx (6.25 copies $/ \mu \mathrm{L})$, as well as rapid point-of-care swab tests, such Quidel Lyra Direct (34 copies/ $\mu \mathrm{L})$, though these were tested using different reference panels and thus direct comparison is difficult ${ }^{18-25}$. Wet testing for cross-reactivity/exclusivity was performed to evaluate potential cross-reactivity/exclusivity of the assay with 21 respiratory pathogens (Fig. 2D, Figure S3). All results, except for the SARS-CoV-2, of wet bench testing were negative (Fig. 2D, Figure S3).

Clinical evaluation of HP-LAMP was performed by comparing results from 65 blinded, paired, nasopharyngeal (NP) swab and saliva samples collected at the same time from symptomatic patients at Jackson Memorial Hospital (JMH) and Columbia University Irving Medical Center (CUIMC). Samples were collected throughout the day without the need for study subjects to be fasting or have previously rinsed their mouths. Samples containing food debris, thick mucus or frank blood were included in the analysis and were not excluded. A representative image of test results of some of the samples collected is shown in Fig. 2E. The testing showed that HP-LAMP had a positive percentage agreement (PPA) of 96.7\% (95\% CI $=82.8-99.9 \%)$ and negative percentage agreement (NPA) of $97.1 \%(95 \% \mathrm{CI}=85.1-99.9 \%)$ (Fig. 2F, Figure S4). The RT-PCR cycle threshold (Ct) values for SARS-CoV-2 target N2 from the NP swab from these positive samples ranged from to 14.2-41.6 (Table S5).

Sample pooling allows multiple people to be tested at once in a single assay. This enables testing of more individuals in a shorter time using fewer resources and is, therefore, an important public health tool ${ }^{26}$. To evaluate the ability of HP-LAMP to be used with pooled samples, we tested pooling of five individual samples. The negative sample matrix was created by individually pooling 80 negative clinical samples into 20 pools of $\mathrm{N}=4$ before adding either a single positive or negative sample to create the final testing pool. Twenty positive pools and 20 negative pools of five were tested by HP-LAMP. HP-LAMP accurately detected 20/20 (100\%) positive pools and 20/20 (100\%) negative pools (Fig. 3).

The equipment costs for performing the HP-LAMP assay are very low; performing the assay requires only a pipette, a mini centrifuge, a vortexer, and two heat blocks that retail for $\sim 250$ USD each. In contrast, the equipment costs for RT-PCR based methods is $>45,000$ USD while the automated Roche cobas 6800 unit costs $\sim 350,000 \mathrm{USD}^{27,28}$. If purchasing the consumable reagents individually using off-the-shelf components, the costs for the HP-LAMP assay is $\sim 80$ USD per assay, and $\sim 16$ USD per assay when pooling five samples. The cost for pre-made HP-LAMP cocktails is 20-25 USD per assay (Sorrento Therapeutics). The consumable cost for RT-PCR based methods is 20-60 USD per assay ${ }^{27}$.

In summary, HP-LAMP enables rapid detection of SARS-CoV-2 directly from saliva without the need for a lab, using a simple, one-step protocol. HP-LAMP has an LoD of $<2$ viral copies per $\mu \mathrm{l}$ of saliva and a positive and negative percentage agreement of $>96 \%$ and $>97 \%$, respectively, compared with RT-PCR testing of paired NP swab samples, comparable to the gold-standard RT-PCR based methods that must be run in a high-complexity laboratory. The simple workflow may also allow adaptation for at-home testing and pooling strategies. An FDA emergency use authorization (EUA) application is currently under review. 


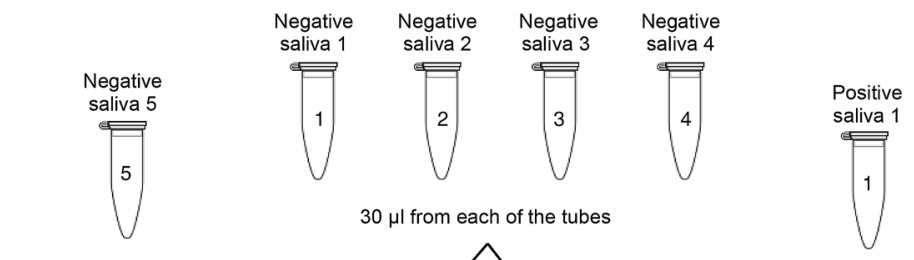

Add $30 \mu \mathrm{l}$ Negative saliva 5

$30 \mu \mathrm{l}$ from each of the tubes
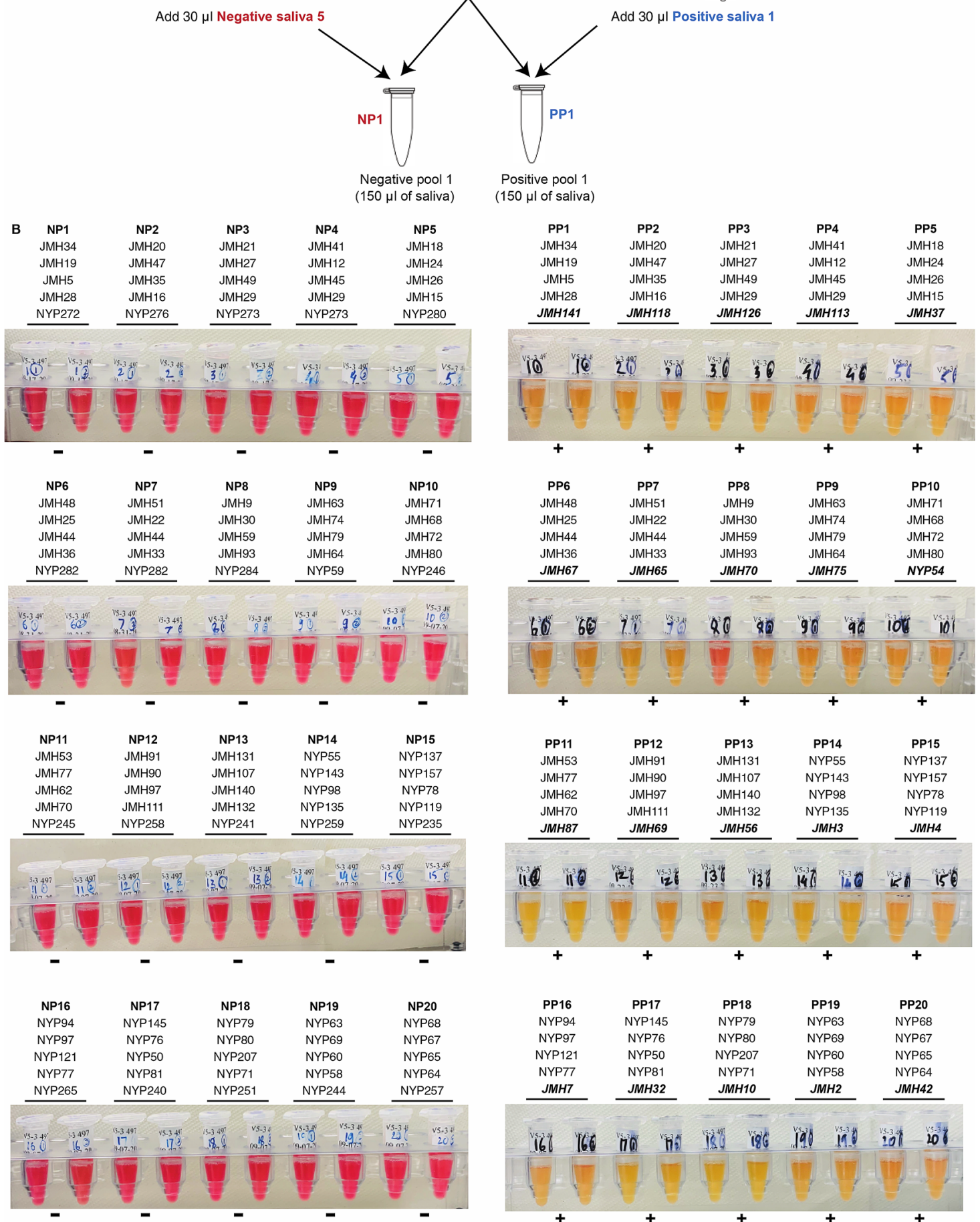

Figure 3. HP-LAMP assay for pooled samples. (A) Illustration of the HP-LAMP assay for sample pooling. A negative matrix was created by pooling 4 known negative saliva samples. A positive or negative pool of 5 samples $(\mathrm{N}=5)$ was created by pooling a known positive or negative sample with the negative matrix. (B) HP-LAMP testing for pooled saliva samples. 20 positive $(n=20)$ and 20 negative $(n=20)$ pools were subjected to HP-LAMP assay. 


\section{Methods and materials}

Ethics. The study was reviewed and approved by the Columbia University Institutional Review Board (IRB) (\#AAAS9893) and all methods were carried out in accordance with relevant guidelines and regulations. All study subjects signed informed consent prior to participating.

Participant enrollment. Study participants were enrolled at New York Presbyterian Hospital when they underwent routine clinical testing for SARS-CoV-2 from 04/29/2020 to 06/1/2020 at the cough and fever clinic or a COVID-19 testing tent. Study participants were enrolled at Jackson Memorial Hospital (JMH) when they underwent routine clinical testing for SARS-CoV-2 upon presentation to the emergency room from 08/14/2020 to $09 / 10 / 2020$.

Sample collection. Nasopharyngeal (NP) swab and saliva samples were obtained from participants following CDC-recommended protocols. Nasopharyngeal swab samples were transported in $3 \mathrm{~mL}$ viral transport medium (VTM) and subjected to routine clinical testing for RT-PCR-based SARS-CoV-2 testing. Saliva samples were self-collected by each participant, by spitting $\sim 1 \mathrm{~mL}$ of saliva into a clean $50 \mathrm{~mL}$ DNA LoBind Conical Tube (Eppendorf, 0,030,122,232). Saliva samples were shipped with $-80^{\circ} \mathrm{C}$ ice packs and stored at $-80^{\circ} \mathrm{C}$ until use. SARS-CoV-2 positive $(n=30)$ and SARS-CoV-2 negative $(n=35)$ samples were included in this study.

Contrived samples for direct saliva testing. Contrived samples were prepared using SARS-CoV-2 Standard $(200,000 \mathrm{cp} / \mathrm{mL}$ ) (Exact Dx, COV019) spike-in or inactivated virus (ATCC, VR-1986HK). SARSCoV-2 RNA Standard was diluted in nuclease-free water (Ambion, AM9937), and 1 to 100 copies of viral RNA were spiked into each reaction along with $5-20 \mu \mathrm{L}$ of saliva from healthy individuals as detailed below. Inactivated virus was spiked into saliva from healthcare workers who tested negative for SARS-CoV-2, and serially diluted to the targeted concentration using additional negative saliva.

Primer design and screening. Primer sets, buffers, and incubation methods were systematically tested to develop the optimized method used herein that would be sufficiently sensitive and robust to enable direct detection of $10^{\circ}$ copies/ $\mu \mathrm{L}$ viral RNA from saliva samples (Figure S1). PrimerExplorer V5 (https://primerexplorer. $\mathrm{jp} / \mathrm{e} /$ ) and the SARS-Cov-2 reference genome NC_045512v2 were used to design the HP-LAMP primers. The primers were matched against human reference genome Hg19 and Human Coronavirus reference genome to ensure specificity 29,30 (Fig. 2B, Figure S1A). Typically, primers for LAMP are designed to target GC-rich regions of the viral RNA because GC-rich regions bind more tightly to primers. However, in SARS-CoV-2, these regions are found towards the $5^{\prime}$ and $3^{\prime}$ ends of the viral RNA. To target the GC-poor (AT-rich) regions in the center portion of SARS-CoV-2 genome, we designed the primers to permit large primer-mediated loop-structures while ensuring that the primers did not form stable secondary structures or self-dimerize. We also aligned the known SARS-CoV-2 genomic sequence with those of six other human coronaviruses (SARS-CoV, MERS-CoV, HCoVHKU-1, HCoV-NL63, HCoV-OC43 and HCoV-229E) to ensure no cross-reactivity.

In-house and published primers ${ }^{12,17}$ were screened using a standard RT-LAMP Protocol (NEB, M1800) (Figure S1A) with the addition of $0.14 \mu \mathrm{M}$ dUTP and $0.0002 \mathrm{unit} / \mu \mathrm{L}$ Antarctic Thermolabile UDG (NEB, M0372S). Primer set V5 was designed to target a central portion of the SARS-CoV-2 genome and was able to detect $10^{\circ}$ copies of viral RNA per reaction with no false positive amplification in the negative control (Figure S1A). It was used for further development of HP-LAMP assay, and is termed as HP-LAMP primer set (Fig. 2B).

The sequences for the primers used are shown in Table S1. CDC 2019-Novel Coronavirus (2019-nCoV) Real-time RT-PCR Primers were also included as a reference ${ }^{31}$.

Preparing one-step RT-LAMP reaction master mix (HP-LAMP). A 25-fold primer mix of LAMP primers (V5.FIP, V5.BIP, V5.LF, V5.LB, V5.F3, V5.B3; Table S1) was prepared by assembling $40 \mu \mathrm{M}$ FIP and BIP, $10 \mu \mathrm{M}$ V5.LF and V5.LB, and $5 \mu \mathrm{M}$ V5.F3 and V5.B3 primers in nuclease-free water (Ambion, AM9937). A $2 \times$ colorimetric RT-LAMP master mix was prepared by adding $3.5 \mu \mathrm{L} 100 \mathrm{mM}$ dUTP (Thermo Scientific, R0133), 0.5 $\mu \mathrm{L}$ Antarctic Thermolabile UDG (NEB, M0372S), and 0.25 $\mathrm{LL} 5 \mathrm{mM}$ SYTO 9 (Invitrogen, S34854) to $1,250 \mu \mathrm{L}$ WarmStart Colorimetric LAMP $2 \times$ Master Mix (DNA \& RNA) (NEB, M1800S/L). The final reaction mix for one reaction includes $250 \mu \mathrm{L} 2 \times$ colorimetric RT-LAMP master mix, $20 \mu \mathrm{L} 25$-fold LAMP primer mix, and $190 \mu \mathrm{L}$ nuclease-free water, $20 \mu \mathrm{L}$ of lysis buffer $((0.1 \%$ tween $-20,2 \%$ volume (i.e., $2 \mu \mathrm{L}$ added to $100 \mu \mathrm{L})$ ezDNase (Invitrogen, 11,766,051)), $0.3 \mathrm{ng} / \mu \mathrm{L}$ lysis buffer volume of carrier DNA (human genomic DNA from a normal male e.g., $6 \mathrm{ng}$ carrier DNA for $20 \mu \mathrm{L}$ lysis buffer), and $\sim 9 \mathrm{ng} / \mu \mathrm{L}$ lysis buffer volume of carrier RNA (NEB, N0362S, $250 \mathrm{ng} / \mu \mathrm{L}$ ), $2 \mu \mathrm{L}$ RNase Inhibitor, Murine (NEB, M0314S/L), 15 $\mu \mathrm{L}$ buffer TE pH 8.0 (Ambion AM9849), and can be scaled up according to the actual number of samples. Lysis buffer was mixed with the carrier gDNA and incubated at RT for $\sim 15$ min before use. For each reaction, $497 \mu \mathrm{L}$ of the final reaction mix was preloaded in a clean $1.5 \mathrm{~mL}$ LoBind microcentrifuge tube (Eppendorf, 022,431,021), stored at $-20^{\circ} \mathrm{C}$, and thawed at $4^{\circ} \mathrm{C}$ before use. This is the final reaction mix used for the HP-LAMP assay, and each sample was tested in duplicate. HP-LAMP assay was QC'ed using negative saliva with $1-2 \times$ LoD inactivated SARS-CoV- 2 virus spike-in, or 25 copies SARS-CoV-2 virus RNA standard.

SARS-CoV-2 detection on saliva samples using HP-LAMP without RNA extraction. Saliva samples were subjected to a $95^{\circ} \mathrm{C}$ heat inactivation for $5 \mathrm{~min}^{32,33}$, and then cooled on ice. $5 \mu \mathrm{L}$ of saliva sample was added to the one-step HP-LAMP final reaction mix, mixed by gentle pipetting using a transfer pipette (Fisherbrand, 13-711-20), and incubated at $63^{\circ} \mathrm{C}$ for $30 \mathrm{~min}$ in a portable heat block (Fisherbrand, 14-955-219). The 
reaction was paused by placing on ice for $1 \mathrm{~min}$, and the colorimetric results were then recorded visually and by camera (Figure S5).

Determining the limit of detection (LoD)-analytical sensitivity. The limit of detection (LoD) is defined as the lowest concentration at which 19/20 replicates (or approximately $95 \%$ of all true positive replicates) are positively detected. To determine the LoD of HP-LAMP, intact SARS-CoV-2 (ATCC VR-1986HK, Batch $70,037,676)$ with a known virus concentration $\left(1.77 \times 10^{5}\right.$ copies/ $\left.\mu \mathrm{l}\right)$ was spiked into saliva from healthcare workers who tested negative for SARS-CoV-2 using the Roche cobas system. The following twofold dilution series was tested: $88.5,44.2,22,11,5.5,2.75,1.38$, and 0.69 copies/ $\mu$ lof saliva. The dilutions of $5.5,2.75,1.38$, and 0.69 copies/ $\mu$ l were tested in triplicate to determine the 'preliminary LoD'. Spiked saliva specimens were tested according to protocol for the HP-LAMP Assay. The preliminary LoD was then confirmed with 20 additional replicates (Figure S2). The LoD of the HP-LAMP Assay was determined to be 1.38 copies/ $\mu$ l of saliva. At this LoD, 19/20 (95\%) individual replicates at a concentration of 1.38 copies/ $\mu$ l of saliva tested positive (Figure S2).

Determining inclusivity (analytical sensitivity). An in silico inclusivity analysis was performed by aligning all primer sequences against all $(n=16,453)$ complete SARS-CoV-2 genomic sequences deposited in the NCBI Virus database on Sep 15, 202015. The HP-LAMP primer set 100\% matched to the ORF1ab gene in $98.8 \%$ of SARS-CoV-2 strains and had 1 mismatch to $1.2 \%$, and 2 mismatches to $0.04 \%$ of strains deposited in the NCBI Virus database, respectively (Table S2).

Determining cross-reactivity (analytical specificity). In silico cross-reactivity was performed by aligning the HP-LAMP primer sequences against sequences of common viruses as well as coronaviruses related to SARS-CoV-2 using NCBI Blast. Both primer FIP and BIP consist of 2 sections of non-continuous genomic sequences and were aligned separately to increase the sensitivity of alignment of cross-reactivity. In total, 6 primers corresponding to 8 sections of virus genome were searched against 32 common pathogens (Table S3) ${ }^{17}$. No pathogen except for SARS-CoV shared $\geq 80 \%$ with the primer sequences. SARS-CoV shared $92.00 \%$ on part 1 ( $~ 50 \%)$ of FIP primer, and $95.80 \%$ on LB primer.

Wet testing was performed to evaluate potential cross-reactivity/exclusivity of the assay with other organisms using ZeptoMetrix Corporation NATtrol Respiratory Verification Panel (ZeptoMetrix, NATRVP-IDI) including 19 respiratory pathogens, NATtrol Coronavirus-SARS Stock (ZeptoMetrix, NATSARS-ST), NATtrol MERSCoV Stock (ZeptoMetrix, NATMERS-ST), and NATtrol SARS-Related Coronavirus 2 (SARS-CoV-2) External Run Control (ZeptoMetrix, NATSARS(COV2)-ERC). Samples were prepared by spiking $3 \mu \mathrm{L}$ inactivated, intact viral particles or bacterial cells using the panels/organisms into negative saliva samples and were subsequently processed using HP-LAMP. Virus and bacteria were tested at concentrations similar to or greater than the SARSCoV-2 virus External Run Control (50,000 copies/mL). All the results of wet bench testing, except for that of SARS-CoV-2, were negative (Table S4, Figure S3).

Clinical evaluation. The performance of HP-LAMP was compared to test results from paired nasopharyngeal (NP) swab samples. The study was conducted with symptomatic patients from Jackson Memorial Hospital (JMH) and Columbia University Irving Medical Center (CUIMC) who each provided a paired NP and saliva sample on the same day. NP samples were immediately processed in the clinical pathology laboratory using FDA authorized Roche $\operatorname{cobas}^{34}$, Cepheid ${ }^{35}$, Qiagen ${ }^{36}$, or EliTech (GendFinder) ${ }^{37}$ systems for SARS-CoV-2 testing at JMH and CUIMC (depending on the available testing option at the time of testing). Saliva was collected in blinded sterile tubes (Eppendorf, 0,030,122,232) without any preservatives and sent to Columbia University Fertility Center for testing by HP-LAMP. A total of 65 samples were tested: 30 samples that were positive for SARS-CoV-2 by NP swab and 35 that were negative by NP swab. After testing, results were sent back to JMH for unblinding (Figure S4). Samples containing food particles or blood were not excluded.

Sample pooling. Sample pooling of 5 samples $(\mathrm{N}=5)$ was performed by combining $30 \mu \mathrm{L}$ from 4 known negative saliva samples with either $30 \mu \mathrm{L}$ of saliva from a positive sample to create a positive pool of 5 samples or $30 \mu \mathrm{L}$ of saliva from a known negative sample to create a negative pool of 5 samples. 20 known positive samples $(\mathrm{N} 2 \mathrm{Ct}<33)$ and 100 known negative samples were used to generate 20 positive pools and 20 negative pools for evaluation of pool testing using HP-LAMP assay.

Received: 10 November 2020; Accepted: 15 February 2021

Published online: 09 March 2021

\section{References}

1. The COVID-19 testing debacle. Nat Biotechnol 38, 653. https://doi.org/10.1038/s41587-020-0575-3 (2020).

2. Weissleder, R., Lee, H., Ko, J. \& Pittet, M. J. COVID-19 diagnostics in context. Sci Transl Med 12, 1931. https://doi.org/10.1126/ scitranslmed.abc1931 (2020).

3. Wyllie, A. L. et al. Saliva or nasopharyngeal swab specimens for detection of SARS-CoV-2. N Engl J Med https://doi.org/10.1056/ NEJMc2016359 (2020).

4. He, X. et al. Temporal dynamics in viral shedding and transmissibility of COVID-19. Nat Med 26, 672-675. https://doi.org/10.1038/ s41591-020-0869-5 (2020). 
5. Lee, S. et al. Clinical course and molecular viral shedding among asymptomatic and symptomatic patients With SARS-CoV-2 infection in a Community Treatment Center in the Republic of Korea. JAMA Intern Med https://doi.org/10.1001/jamaintern med.2020.3862 (2020)

6. Lalli, M. A. et al. Rapid and extraction-free detection of SARS-CoV-2 from saliva by colorimetric reverse-transcription loopmediated isothermal amplification. Clin Chem https://doi.org/10.1093/clinchem/hvaa267 (2020).

7. L'Helgouach, N. et al. EasyCOV: LAMP based rapid detection of SARS-CoV-2 in saliva. medRxiv, 2020.2005.2030.2011729120112020.20117205.20117230.20117291 (2020).

8. Vogels, C. B. F. et al. SalivaDirect: A simplified and flexible platform to enhance SARS-CoV-2 testing capacity. medRxiv pre-print, 2020.2008.2003.20167791-20162020.20167708.20167703.20167791 (2020).

9. Rabe, B. A. \& Cepko, C. SARS-CoV-2 detection using isothermal amplification and a rapid, inexpensive protocol for sample inactivation and purification. Proc. Natl. Acad. Sci. 117, 24450-24458. https://doi.org/10.1073/pnas.2011221117 (2020).

10. Smyrlaki, I. et al. Massive and rapid COVID-19 testing is feasible by extraction-free SARS-CoV-2 RT-PCR. Nat. Commun. 11, 4812-4812. https://doi.org/10.1038/s41467-020-18611-5 (2020).

11. Notomi, T. Loop-mediated isothermal amplification of DNA. Nucleic Acids Res. 28, 63e-663. https://doi.org/10.1093/nar/28.12. e63 (2000)

12. Zhang, Y. et al. Rapid molecular detection of SARS-CoV-2 (COVID-19) virus RNA using colorimetric LAMP. medRxiv, 2020.2002.2026.20028373-20022020.20028302.20028326.20028373, doi:https://doi.org/10.1101/2020.02.26.20028373 (2020).

13. Lamb, L. E., Bartolone, S. N., Ward, E. \& Chancellor, M. B. Rapid detection of novel coronavirus/Severe Acute Respiratory Syndrome Coronavirus 2 (SARS-CoV-2) by reverse transcription-loop-mediated isothermal amplification. PLoS ONE 15, e0234682. https://doi.org/10.1371/journal.pone.0234682 (2020).

14. Yu, L. et al. Rapid detection of COVID-19 Coronavirus using a reverse transcriptional loop-mediated isothermal amplification (RT-LAMP) Diagnostic Platform. Clin Chem 66, 975-977. https://doi.org/10.1093/clinchem/hvaa102 (2020).

15. Brister, J. R., Ako-Adjei, D., Bao, Y. \& Blinkova, O. NCBI viral genomes resource. Nucleic Acids Res 43, D571-577. https://doi. org/10.1093/nar/gku1207 (2015).

16. Fernandes, J. D. et al. The UCSC SARS-CoV-2 Genome Browser. Nat Genet 52, 991-998. https://doi.org/10.1038/s41588-0200700-8 (2020).

17. Wei, S. et al. Direct diagnostic testing of SARS-CoV-2 without the need for prior RNA extraction. Sci. Rep. 11, 2402. https://doi. org/10.1038/s41598-021-81487-y (2021).

18. Centers for Disease Control and Prevention. CDC 2019-Novel Coronavirus (2019-nCoV) Real-Time RT-PCR Diagnostic Panel Instruction for Use. 3, 0-0 (2020).

19. Laboratory Corporation of America (LabCorp). COVID-19 RT-PCR test EUA Summary. Coronavirus Disease 2019 (COVID-19) Emergency Use Authorizations for Medical Devices, 1-29 (2020).

20. U.S. Food \& Drug Administration. SARS-CoV-2 Reference Panel Comparative Data. https://www.fda.gov/medical-devices/coron avirus-covid-19-and-medical-devices/sars-cov-2-reference-panel-comparative-data (2020, October 7).

21. Yale School of Public Health Department of Epidemiology of Microbial, D. SalivaDirect assay EUA Summary (2020).

22. Fluidigm Corporation. Advanta Dx SARS-CoV-2 RT-PCR Assay Instructions for Use. Coronavirus Disease 2019 (COVID-19) Emergency Use Authorizations for Medical Devices (2020).

23. Abbott Diagnostics Scarborough, Inc. Id Now ${ }^{\text {ma }}$ Covid-19 Instructions for Use. Coronavirus Disease 2019 (COVID-19) Emergency Use Authorizations for Medical Devices, 1-17 (2020).

24. Quidel Corporation. Lyra ${ }^{\circledR}$ SARS-CoV-2 Assay Instructions for Use. Coronavirus Disease 2019 (COVID-19) Emergency Use Authorizations for Medical Devices 1, 1-21 (2020).

25. Quadrant Biosciences Inc. Clarifi COVID-19 Test Kit Instructions for Use. Coronavirus Disease 2019 (COVID-19) Emergency Use Authorizations for Medical Devices.

26. U.S. Food \& Drug Administration. Coronavirus (COVID-19) Update: FDA Issues First Emergency Authorization for Sample Pooling in Diagnostic Testing. https://www.fda.gov/news-events/press-announcements/coronavirus-covid-19-update-fda-issues-first -emergency-authorization-sample-pooling-diagnostic (2020, July 18).

27. Chow, F. W. et al. A rapid, simple, inexpensive, and mobile colorimetric assay COVID-19-LAMP for mass on-site screening of COVID-19. Int J Mol Sci 21, 5380. https://doi.org/10.3390/ijms21155380 (2020).

28. National Institutes of Health. LAB SUPPLIES CPT CT NG HPV FOR USE ON ROCHE 6800. https://projects.propublica.org/coron avirus-contracts/contracts/V797D70105 (2020).

29. Hatcher, E. L. et al. Virus variation resource-improved response to emergent viral outbreaks. Nucleic Acids Res 45, D482-D490. https://doi.org/10.1093/nar/gkw1065 (2017).

30. Kent, W. J. et al. The human genome browser at UCSC. Genome Res. 12, 996-1006. https://doi.org/10.1101/gr.229102 (2002).

31. Centers for Disease Control and Prevention. Research use only 2019-novel coronavirus (2019-nCoV) real-time RT-PCR primers and probes. https://www.cdc.gov/coronavirus/2019-ncov/lab/rt-pcr-panel-primer-probes.html (2020).

32. Batéjat, C., Grassin, Q., Manuguerra, J.-C. \& Leclercq, I. Heat inactivation of the Severe Acute Respiratory Syndrome Coronavirus 2. bioRxiv, 2020.2005.2001.067769-062020.067705.067701.067769 (2020).

33. Fomsgaard, A. S. \& Rosenstierne, M. W. An alternative workflow for molecular detection of SARS-CoV-2-escape from the NA extraction kit-shortage, Copenhagen, Denmark, March 2020. Eurosurveillance 25, 1-8. https://doi.org/10.2807/1560-7917. ES.2020.25.14.2000398 (2020).

34. Roche Molecular Systems, Inc. cobas SARS-CoV-2 Instructions for Use. (Coronavirus Disease 2019 (COVID-19) Emergency Use Authorizations for Medical Devices, 2020).

35. Cepheid. Xpert ${ }^{\circledR}$ Xpress SARS-CoV-2 Instructions for Use. Coronavirus Disease 2019 (COVID-19) Emergency Use Authorizations for Medical Devices (2020).

36. QIAGEN GmbH. QIAstat-Dx Respiratory SARS-CoV-2 Panel Instructions for Use (Handbook). Coronavirus Disease 2019 (COVID19) Emergency Use Authorizations for Medical Devices, 1-120 (2020).

37. Osang Healthcare. GeneFinder ${ }^{\text {TM }}$ COVID-19 PLUS Real Amp Kit Instructions for Use. Coronavirus Disease 2019 (COVID-19) Emergency Use Authorizations for Medical Devices 2020, 1-10 (2020).

\section{Acknowledgements}

We thank Dr. Mary D'Alton, Michelle DiVito, and members of Columbia University Fertility Center, the Department of Obstetrics and Gynecology, Dr. Steven Spitalnik of the Department of Pathology and Cell Biology, the Suh Laboratory, Clinical Microbiology Laboratory, Columbia University Laboratory of Personalized Genomic Medicine at Columbia University Medical Center and New York Presbyterian Hospital, and the University of Miami, for their helpful input and support for this study. This study is supported by NIH grants R01HD100013 (Z.W.), R01HD086327 (Z.W.), 1RF1AG057341 (Y.S), and R01AG057433 (Y.S) and the John and Wendy Havens Innovators Program. 


\section{Author contributions}

Study conception and design: Z.W. and S.W.; experiments: S.W., H.S., A.D., E.K.; All co-authors participated in study design, patient recruitment, data interpretation and study coordination. S.W., H.S., A.D, and Z.W., wrote the main manuscript. All authors reviewed and edited the manuscript.

\section{Competing interests}

Columbia University has filed patent applications relating to this technology with Drs. Wei and Williams listed as inventors. Columbia University has entered into a licensing agreement relating to this technology with Sorrento Therapeutics. Drs. Williams is a consultant to Sorrento Therapeutics. Other authors declare no competing interests.

\section{Additional information}

Supplementary Information The online version contains supplementary material available at https://doi. org/10.1038/s41598-021-84792-8.

Correspondence and requests for materials should be addressed to Z.W.

Reprints and permissions information is available at www.nature.com/reprints.

Publisher's note Springer Nature remains neutral with regard to jurisdictional claims in published maps and institutional affiliations.

(c) (i) Open Access This article is licensed under a Creative Commons Attribution 4.0 International License, which permits use, sharing, adaptation, distribution and reproduction in any medium or format, as long as you give appropriate credit to the original author(s) and the source, provide a link to the Creative Commons licence, and indicate if changes were made. The images or other third party material in this article are included in the article's Creative Commons licence, unless indicated otherwise in a credit line to the material. If material is not included in the article's Creative Commons licence and your intended use is not permitted by statutory regulation or exceeds the permitted use, you will need to obtain permission directly from the copyright holder. To view a copy of this licence, visit http://creativecommons.org/licenses/by/4.0/.

(C) The Author(s) 2021 